\title{
Cognitive Human Resource Allocation Mechanism based on Game Theory
}

\author{
Yu Bo \\ Xijing University, Xi'an Shaanxi, 710123, China \\ 13201585562@yeah.net
}

\begin{abstract}
To overcome the "prematurity" of standard particle swarm optimization in scheduling problem for solution to resource constrained project, an improved cultural particle swarm optimization is proposed. The algorithm framework is based on the main group space of particle swarm optimization and the knowledge space of cultural algorithm, and both spaces have their own spaces and conduct independent and parallel evolvement to form a "dual evolvement and dual promotion" mechanism and increase the global searching ability and operation efficiency of the algorithm. Meanwhile, to avoid the restriction of self-evolvement for the knowledge space of cultural algorithm, the evolvement mechanism of genetic algorithm is introduced to improve the evolvement operation of knowledge space. Finally, the effectiveness of improved cultural particle swarm optimization in solving the problem of resource constrained project can be validated through comparison of example of human resource scheduling.
\end{abstract}

Keywords: Cultural particle swarm optimization; Resource scheduling; Human resource; Knowledge space; Main group

\section{Introduction}

Project Scheduling Problem for Resource Constrained, PSP4RC is to minimize the total construction period through arranging the commencing time for all tasks of the project under the premise of satisfying the preceding constraint and resource constraint. PSP4RC is a typical NP-hard problem [1] which has attracted much attention since 1950s, and many scholars conduct a great amount of research on PSP4RC with many results. The solution methods of PSP4RC range from accurate solution to satisfactory solution; from linear programming, nonlinear programming, branch and bound approach [2] to the current heuristic algorithm, including genetic algorithm [3-4], simulated annealing algorithm [5-6], particle swarm optimization [7-8], ant colony optimization [9], etc., among which particle swarm optimization is the relatively successful swarm intelligence algorithm applied in PSP4RC. However, the traditional particle swarm optimization tends to fall into local optimization and results in the phenomenon of "prematurity" due to its own algorithm constraint in solving PSP4RC problem which will cause certain difference between the optimized result and the ideal result. Cultural algorithm is a calculation model based on the multi-evolvement process of groups, and any evolutionary algorithm conforming to the requirements of cultural algorithm can be embedded into the framework of cultural algorithm. In addition, the constraint of self-evolvement mechanism for knowledge space of cultural algorithm results in unsatisfactory result for knowledge space group during self-evolvement, reduced effective influence on lower main group space and affected global searching ability and operation efficiency of the whole algorithm. Based on the above, the Improved Cultural Particle Optimization is put forward in the thesis based on the framework of cultural algorithm, and the selfevolvement mechanism of knowledge space is improved through species evolvement of genetic algorithm with a view to reducing the probability of early reduction into local 
optimization of particles during the solution of PSP4RC problem and better solving the PSP4RC problem.

\section{Game Description}

Although capacity expansion can increase the operation income of public resource library, it can also make the supplier undertake the corresponding cost. Therefore, the capacity expansion strategy for public resource library of the supplier must be optimized. The capacity expansion theory can be defined as $\left\langle\square,\left\{\mathrm{T}_{s},\right\},\left\{u_{s}(\cdot)\right\}\right\rangle$, and then the balance strategy modeling and solution will be conducted. $\square$ means a group of players (i.e. supplier). The strategy is the capacity to be expanded (i.e. reserved bandwidth and server). Suppose the strategy space of the supplier as a discrete set $\mathrm{T}_{\mathrm{s}}=\left\{t_{s}=\left(K_{b, s}^{b w}(i), K_{d, s}^{c p}(i)\right) ; i \in\left\{1, \cdots, I_{s}\right\}\right\}$ among which $I_{s}$ is the selected total number for capacity expansion of the supplier $s$. It is required to point out that $K_{b, s}^{b w}(i=1)=K_{b, s}^{b w}$ and $K_{d, s}^{c p}(i=1)=K_{d, s}^{c p}$ respectively indicate the initial reserved bandwidth and server. The income of the supplier can be defined as $u_{s}\left(t_{s}, t_{-s}\right)=\mu_{s}\left[v(\square),\left(t_{s}, t_{-s}\right)\right]-C_{s}(i)$ among which $t_{s}$ indicates the strategy of the supplier $S$ and $t_{-s}$ indicates the strategies of other suppliers expect the supplier $s$. At this time, all the dual incomes $u_{s}(\cdot)$ calculated by the supplier $S$ based on the formula (15) can be defined as the function for capacity expansion strategy of all suppliers (i.e. $t_{s}$ and $\left.t_{-s}\right) . C_{s}(i)$ is the fixed cost borne by the supplier $s$ during implementation of expansion strategy $i$. It is required to point out that the access control and income distribution make the resource capacity results of capacity expansion game as the input. Meanwhile, to get the solution of capacity expansion game, the dual incomes of access control and income distribution are required.

Suppose that the single project includes $J$ jobs $(j=1,2, \ldots, J), S T_{j}$ is the beginning time of job $j$, and $d_{j}$ is the execution time of job $j\left(j=1,2, \ldots, K, k\right.$ is the number of jobs); $r_{j k}^{p}$ is the required quantity of job $j$ for the renewable resources and $R_{k}^{p}$ is the total quantity of the $k$ resource $\left(k=1,2, \ldots, K, K\right.$ is number of types of renewable resources); $p_{j}$ is the preceding working set of job $j$; the mathematical model of the problem is:

$$
\begin{aligned}
& \min f=S T_{j} \\
& \text { s.t. } \quad S T_{j}-S T_{i} \geq d_{j}, i \in P_{i} \\
& \sum_{j \in A_{t}} r_{j k}^{p} \leq R_{k}^{p}, t=1,2, \cdots, S T_{j}
\end{aligned}
$$

Where: the formula (1) is the target function which indicates that the commencing time for the last job $J$ is the minimum, i.e., the total construction period of the project is the minimum; the formula (2) is the temporal constraint, i.e., the preceding relationship constraint which indicates that the job $j$ cannot be started until the job $I$ is finished for any job $i \in P_{j}$; the formula (3) is the resource constraint which indicates that the job cannot be interrupted once started, and the application quantity of various resources at any time cannot be greater than the available quantity. 


\section{Algorithm Description}

\subsection{Particle Swarm Optimization and Cultural Algorithm}

Suppose that in a $D$-dimensional target searching space, there is a group composed of $N$ particles, and the position coordinate for $i(i=1,2, \ldots, N)$ particle of $t$ generation is indicated as $x_{i}^{t}=\left(x_{i 1}, x_{i 2}, \cdots, x_{i d}, \cdots, x_{i D}\right)^{T}$, the speed can be indicated as: $v_{i}^{t}=\left(v_{i 1}^{t}, v_{i 2}^{t}, \cdots, v_{i d}^{t}, \cdots, v_{i D}^{t}\right)^{T}$, the optimal position for the individual can be indicated as: $p_{i}^{t}=\left(p_{i 1}^{t}, p_{i 2}^{t}, \cdots, p_{i d}^{t}, \cdots, p_{i D}^{t}\right)^{T}$, and the global optimization of the group can be indicated as: $p_{g}^{t}=\left(p_{g 1}^{t}, p_{g 2}^{t}, \cdots, p_{g d}^{t}, \cdots, p_{g D}^{t}\right)^{T}$.

The $d$-dimensional $i$ particle of $t+1$ generation can be updated according to the following formulas:

$v_{i d}^{t+1}=\omega v_{i d}^{t}+c_{1} r_{1}\left(p_{i d}^{t}-x_{i d}^{t}\right)+c_{2} r_{2}\left(p_{g d}^{t}-x_{i d}^{t}\right)$

$x_{i d}^{t+1}=x_{i d}^{t}+v_{i d}^{t+1}$

Where, $\omega$ is the inertia weight which controls the influence of the previous speed on the current speed. The learning factors of $c 1$ and $c 2$ adjust the weight for influence of own experience and social (group) experience of particles on the activity, and $r 1$ and $r 2$ are the random numbers evenly distributed in the scope of $(0,1)$

The cultural algorithm provides an explicit mechanism to obtain, save and integrate the knowledge and experience for optimizing and solving of microscopic evolvement group. The main group space is the main space for problem solving of algorithm operation, and the self iterative solving is conducted through evolvement operation and performance evaluation; the knowledge information constantly produced through main group space is saved into the knowledge space through the reception operation, and the knowledge space is updated through its own evolvement which guides through affecting further evolvement for main group space of the operation. The cultural algorithm is of the characteristics such as dual evolvement and inheritance, support of the self-adaptive evolvement of two spaces and support of species evolvement mechanism for different algorithms. Refer to Figure 1 for the procedure of the cultural algorithm.

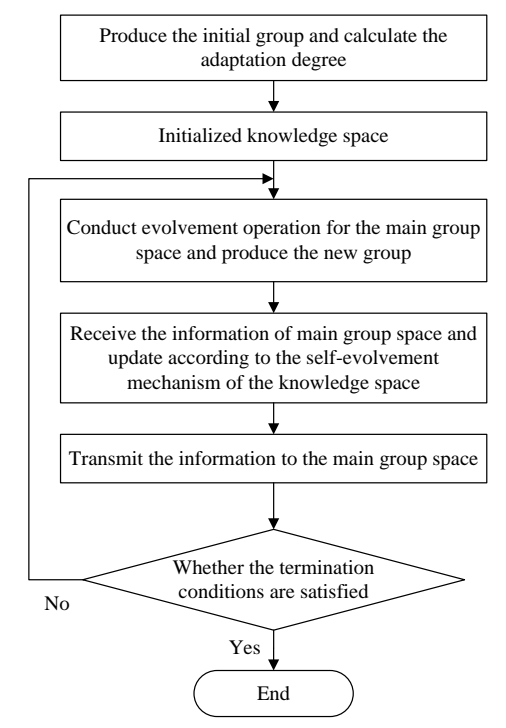

Figure 1. Flow Chart of Cultural Algorithm 


\subsection{Improved Cultural Particle Optimization}

3.2.1. The Procedure for Improved Cultural Particle Optimization: Refer to Figure 2, for the procedure of Improved Cultural Particle Optimization

Step 1: The parameters for initialized main group space and knowledge space such as maximum iterations $G 1$ and $G 2$, species size, inertia weight and acceleration constant.

Step 2: calculate the adaptation degree value of each particle, save the initial best position and the initial optimal adaptation degree value in each group.

Step 3: calculate the new speeds for all particles of knowledge space, calculate the new positions of all particles according to formula (4) and formula (5), and conduct amplitude limiting for new positions and speeds of all particles; calculate the new speeds for all particles of group space, calculate the new positions of all particles and conduct amplitude limiting for new positions and speeds of all particles.

Step 4: Update the individual historical best adaptation degree and individual historical best position of all particles; update the group historical best adaptation degree and group historical best position of all groups.

Step 5: judge whether it is true through receiver function, and if it is true, that is exceeding the continuously unimproved algebra for optimal adaptation degree of particle in the whole group for allowable group space and knowledge space, the particle with the optimal adaptation degree of group space will replace the particle with the worst adaptation degree of the knowledge space.

Step 6: if the stop conditions are satisfied, namely the iterations exceed the maximum allowable iterations or the searched optimal position satisfies the reserved minimum adaptive threshold, the search will be stopped and the optimal position and optimal adaptive value be output; otherwise, return to Step 3 to continue to search.

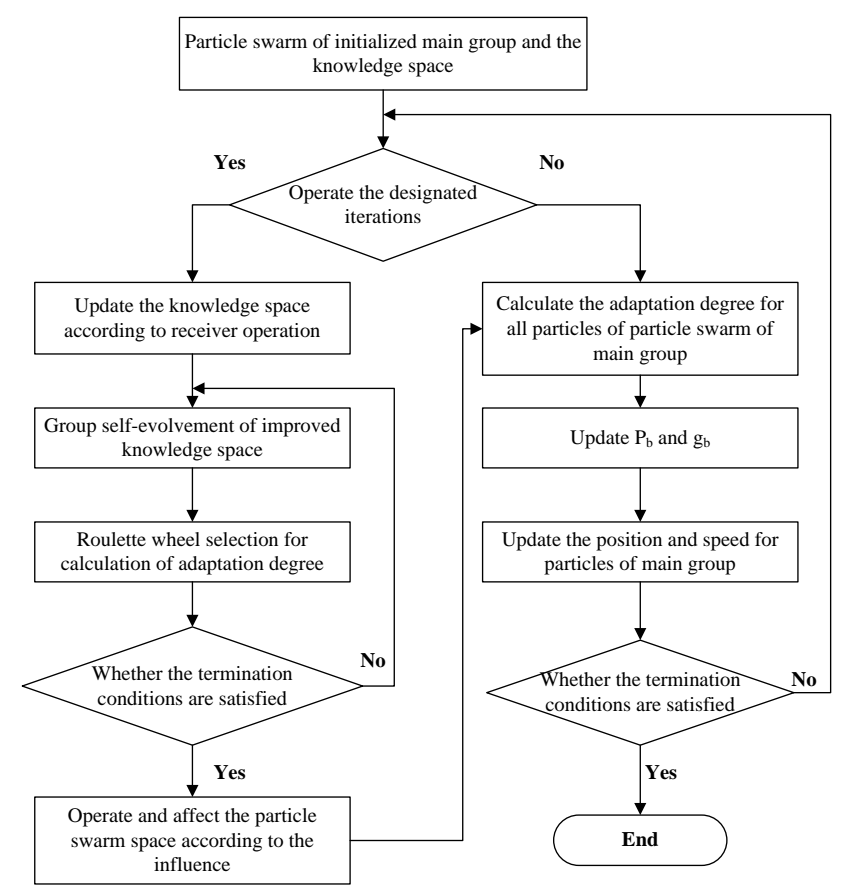

Figure 2. Flow Chart of ICPSO Algorithm

3.2.2. Self-Evolvement Operation for Improved Knowledge Space Group: The selfevolvement and update of knowledge space group is improved through the evolvement 
mechanism of genetic algorithm, and the selection operation, crossover operation and mutation operation will be adopted for self-evolvement.

\section{(1) Selection operation}

The species scale is $n$. Before crossover operation, the parent individual must be selected from the current species through selection operation. The procedure for selection operation algorithm of crossover operation to select individual is as follows:

Step1: calculate $f(\mathrm{i}), i=1, \ldots, n$ for all the individuals of current species.

Step2: determine the selection probability $P\left(C_{i}\right)$ for all individuals. In the proportional selection method, the probability for the excellent individual to be chosen is higher than that for the inferior individual which will result in the rapidly shortened searching scope and can easily cause the prematurity of premature convergence, so the probability to be chosen for individual will be determined based on the method of sequencing selection in the thesis which refers to that the descending order will be conducted according to the size of adaptation degree with the individual after sequencing as $\{\mathrm{C} 1, \mathrm{C} 2, \ldots, \mathrm{Cn}\}$. The probability for the individual to be chosen is:

$P\left(C_{i}\right)=\frac{1}{n}\left\{\sigma^{g}-\frac{\sigma^{g}-\sigma^{b}}{n-1}(i-1)\right\}$

In the above formula, $i$ is the serial number of individual, $\sigma^{g}$ is the expected value of the optimal individual $\mathrm{C} 1$ after selection operation, and $\sigma^{b}$ is the expected value of the worst individual $\mathrm{Cn}$ after selection operation, $\sigma^{b}=n \times P\left(C_{n}\right)$. It is generally required that $1 \leq \sigma^{g} \leq 2$ and $\sigma^{b}=2-\sigma^{g}$. When $\sigma^{g}=2$ and $\sigma^{b}=0$, the expected number for the worst individual in the survival of next generation is 0 , and the probability for the optimal individual to be chosen is higher than that for other individuals which makes premature convergence of algorithm easier; when $\sigma^{g}=\sigma^{b}=1$, the selection method is changed into evenly distributed random selection. In general, $\sigma^{g}=1.1$ can be selected.

Step3: the roulette wheel selection is adopted to save the relatively excellent individual according to the individual probability calculated in formula (6).

\section{(2) Crossover operation}

The single-point crossover method is adopted in the thesis. For the selected two individuals, randomly select one crossover point and then exchange the priority values after the point as shown in Figure 3.

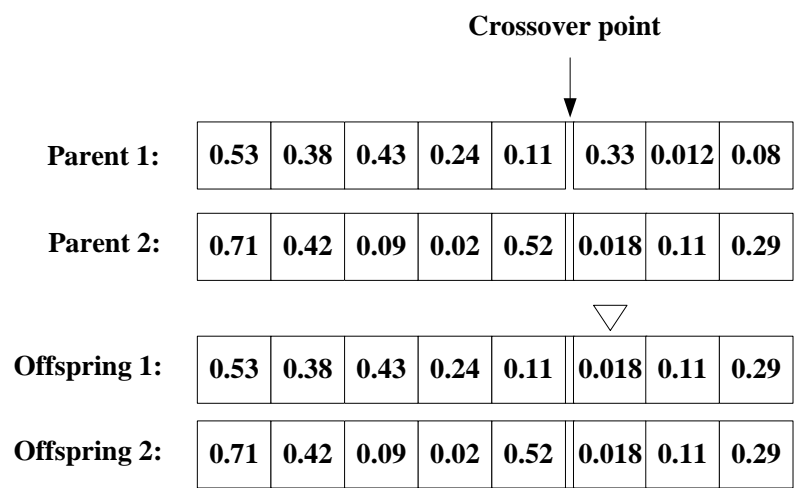

Figure 3. Crossover Operation 


\section{(3) Mutation Operation}

Mutation operator operates on the individual through mutation probability $\mathrm{Pm}$. Select some priority value of some individual according to the mutation probability $P m$, and replace the original value with the evenly distributed random value in the interval of $(0,1)$.

\section{PSP4RC Problem Solving}

\subsection{Encoding Design}

The encoding scheme based on priority value is adopted in the thesis, and a priority sequence is randomly generated in the scope of $(0,1)$ to make each activity have a sole priority. In PSP4RC model, one feasible solution is represented by one particle. Each particle serves as one point of $D$-dimensional space among which $D$ elements represent the $D$ activities of the PSP4RC problem to be solved. Therefore, $D$-dimensional parameter $C_{i}=\left\{C_{i 1}, C_{i 2}, \cdots, C_{i D}\right\}$ indicates the $D$-dimensional position of particle $i$ and represents the priority value of $D$ activities as shown in Figure 4.

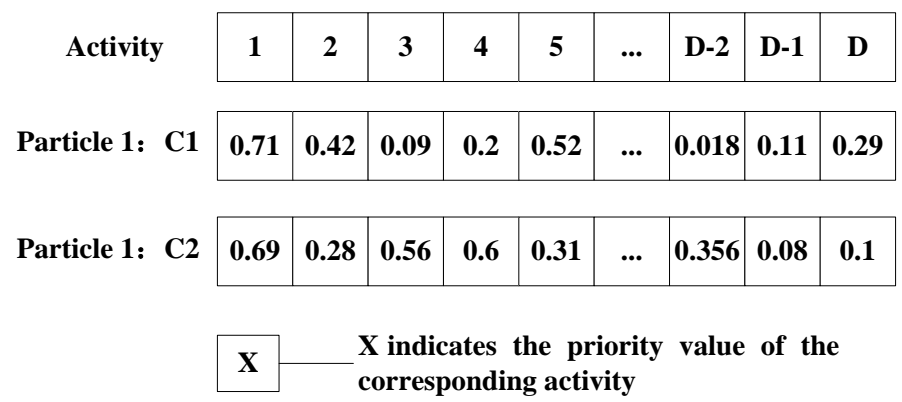

Figure 4. Priority Value of Particle

\subsection{Generation of Scheduling Scheme}

The serial scheduling scheme will be adopted as the decoding rule. The target value of PSP4RC problem is the minimum construction period which is calculated according to the generated scheduling sequence of job and the resource constraint, and the specific solving procedure is as follows.

Step1: in the initialized resource list, the initialized scheduling number $I D$ equals to 1 , and suppose $k=1$;

Step2: Extract the working serial number $S k$ in scheduling list, offer $S k$ to $I D$, search all the preceding job for job $I D$, and select the ending time of the latest completion job as the earliest beginning time $S T I D$ of job $I D$.

Step3: judge whether all the resources conform to the requirements, and if the requirements are satisfied, turn to Step 5; otherwise, turn to Step 4;

Step4: delay the earliest commencing time by one day, i.e., $S T I D=S T I D+1$, then recalculate the supply quantity of resource, and turn to Step 3;

Step5: calculate the completion time FTID of job ID according to duration of job, adopt the resource distribution method of preemptive mode, deduct the used resource from the resource list and then update the resource list;

Step6: if $k<J$ and $k=k+1$, turn to Step 2; otherwise the obtained earliest commencing time of job $J$ is the target value - construction period of the project. 


\section{Example Solving and Result Analysis}

The examples of the thesis are from the documents [13-14]. The network planning technology is adopted in document [13] and the particle swarm optimization algorithm is adopted in document [14] to solve the resource-constrained minimum construction period of the example. According to the network planning diagram after encoding under simple encoding rule as shown in Figure 5, the project includes 9 actual activities, the Quantity limitation for resources of the project is 12 humans and the inform detail for the project is shown in Table 1.

Table 1. Detailed Statement of Project Information

\begin{tabular}{ccccc}
\hline Activity & $\begin{array}{c}\text { Resource } \\
\text { /manpower }\end{array}$ & $\begin{array}{c}\text { Construction } \\
\text { period/days }\end{array}$ & Preceding activity & $\begin{array}{c}\text { Subsequent } \\
\text { activity }\end{array}$ \\
\hline 1 & - & - & - & $2,3,4$ \\
2 & 6 & 2 & 1 & 5,6 \\
3 & 3 & 4 & 1 & 7 \\
4 & 5 & 5 & 1 & 8,9 \\
5 & 4 & 4 & 2 & 8,9 \\
6 & 7 & 3 & 2 & 10 \\
7 & 4 & 7 & 3 & 11 \\
8 & 5 & 6 & 4,5 & 10 \\
9 & 3 & 4 & 4,5 & 11 \\
10 & 5 & 2 & 6,8 & 11 \\
11 & - & - & $7,9,10$ & - \\
\hline
\end{tabular}

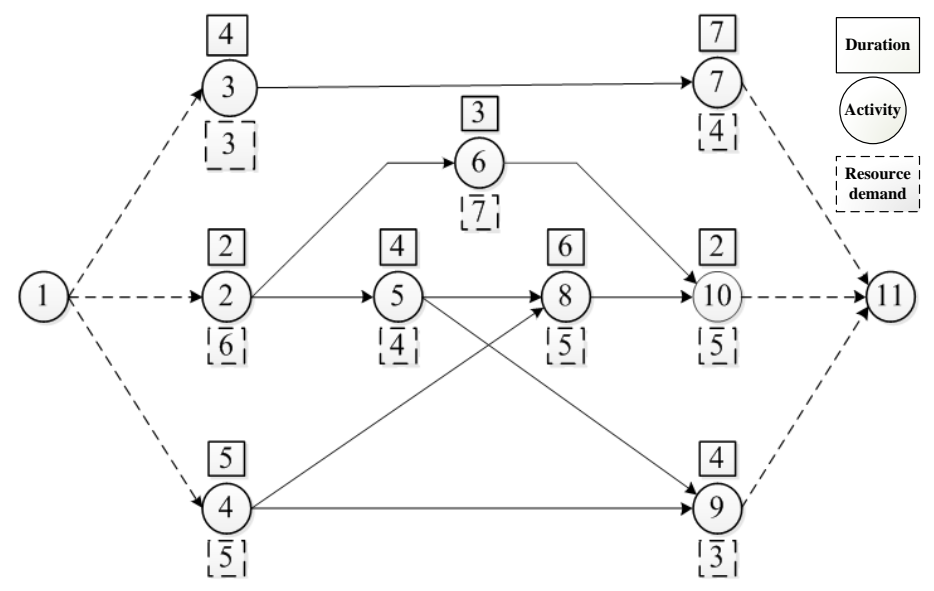

Figure 5. Network Diagram for Code of Project List

Parameter setting: the species scale if 50, the iterations are 200, inertia factor $\omega$ is 1.2 , $C 1$ and $C 2$ are 2, the solution space of knowledge is $40 \%$ of the species scale, the Project VBA programming is adopted and the operation time is 100 .

In the above example, the maximum satisfactory solution (minimum construction period) for solving probability of the thesis is 16 which is less than the corresponding result (minimum construction period of 17 days) of documents [13-14] by one day and is much excellent. It is indicated that to solve the Project Scheduling Problem for Resource Constrained, the cultural particle swarm optimization has certain advantages in expanding the searching scope and avoid the "prematurity" compared with the above solving method. 


\section{Table 2. Corresponding Scheduling Scheme for Maximum Probability} Solution (16 Days)

\begin{tabular}{cccccc}
\hline \multirow{2}{*}{ Activity } & $\begin{array}{c}\text { Construction } \\
\text { Period }\end{array}$ & $\begin{array}{c}\text { Optimization scheme 1 } \\
\text { Commencing } \\
\text { time }\end{array}$ & $\begin{array}{c}\text { Ending } \\
\text { time }\end{array}$ & $\begin{array}{c}\text { Optimization scheme 2 } \\
\text { timencing }\end{array}$ & $\begin{array}{c}\text { Ending } \\
\text { time }\end{array}$ \\
\hline 1 & 0 & Virtual job & Virtual job & Virtual job & Virtual job \\
2 & 2 & 1 & 2 & 1 & 2 \\
3 & 4 & 3 & 6 & 1 & 4 \\
4 & 5 & 1 & 5 & 3 & 7 \\
5 & 4 & 3 & 6 & 3 & 6 \\
6 & 3 & 7 & 9 & 7 & 9 \\
7 & 7 & 10 & 16 & 10 & 16 \\
8 & 6 & 7 & 12 & 8 & 13 \\
9 & 4 & 10 & 13 & 10 & 13 \\
10 & 2 & 13 & 14 & 14 & 15 \\
11 & 0 & Virtual job & Virtual job & Virtual job & Virtual job \\
\hline
\end{tabular}

In the solutions of calculation examples described above, the satisfactory solution (minimum construction period) is improved than that in relative document but also more project scheduling schemes are found so that global searching ability of algorithm and species diversity are expressed, which is shown in Table 2. It shows that Cultural Particle Optimization has rather better practical significance on scheduling problem of project with limited resource. In practical project, it can provide more decision schemes and choices for decision maker.

After program runs for 100 times, collect the categories of satisfactory solution (minimum construction period) in each operation and their corresponding scheduling schemes and times of each satisfactory solution. For the program with shorter construction period, Cultural Particle Optimization has greater percentage $(82 \%)$ to get satisfactory solution in comparison with relative results of document [14], which is shown in Fig.6. It shows that Cultural Particle Optimization has better capability than Standard Particle Swarm Optimization in optimizing, and it enables scheduling problem of project with limited resource to get best satisfactory solution with greater percentage and dramatically reduces the cost and burden in solving relative problems in reality.

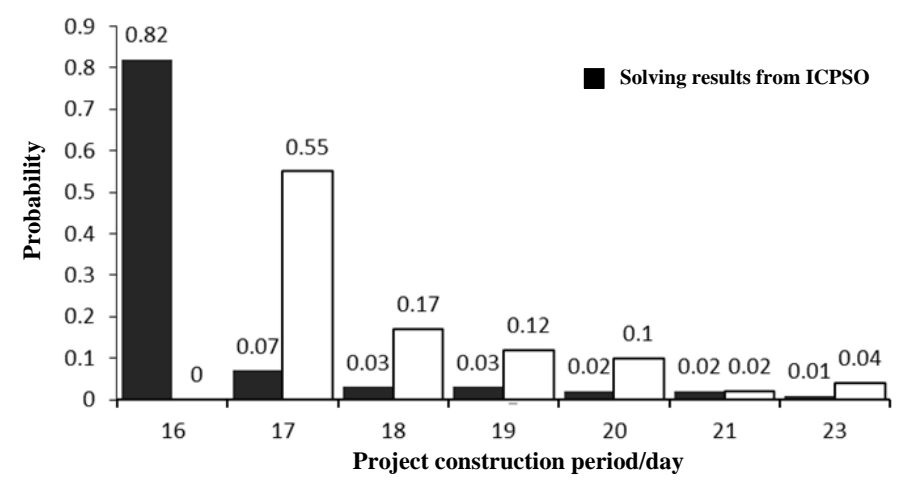

Figure 6. Project Duration and Probability of Running 100 Times

Network scheme technical and Standard Particle Swarm Optimization, genetic algorithm and Improved Cultural Particle Optimization are respectively used to solve the examples described above. Among them, $d \max , d \min$ and $d$ mean are the maximum value, minimum value and mean value of the deviation between algorithm solution and optimization solution. ${ }^{\omega}$ is the proportion of optimization solution in all solutions and 
$d$ time is the mean calculation time of algorithm. Calculation result is shown in Table.3. Among the four algorithms, ICPSO algorithm has the best accuracy and gets the optimization solution in greater percentage; because iterative evolution of two group spaces is necessary so that calculation duration of ICPSO is little longer than GA and PSO. Generally, ICPSO algorithm has better results on scheduling problem of project with limited resource with its solving efficiency and performance better than GA and PSO.

Table 3. Comparison of Calculation Results for Algorithm

\begin{tabular}{cccccc}
\hline Algorithm & $d_{\min }$ & $d_{\max }$ & $d_{\text {mean }}$ & $\omega$ & $d_{\text {time }}$ \\
\hline CPM[13] & 0 & 3 & 2 & $60 \%$ & 30 \\
GA[14] & 1 & 6 & 2.3 & $55 \%$ & 6 \\
PSO & 0 & 4 & 1.9 & $75 \%$ & 9 \\
ICPSO & 0 & 1 & 0.3 & $82 \%$ & 11 \\
\hline
\end{tabular}

\section{Conclusion}

The Improved Cultural Particle Optimization is proposed in the thesis based on the Project Scheduling Problem for Resource Constrained. The main group space and knowledge space of the algorithm independently evolve and form a "dual evolvement and dual promotion" mechanism which strengthens the species diversity of algorithm; the species evolvement mechanism of genetic algorithm can be used to improve the selfevolvement mechanism of the knowledge space, strengthen the global searching ability during species evolvement, better avoid the reduction into local optimizing and strengthen the optimizing efficiency. It is proved that compared with other algorithms, the Improved Cultural Particle Optimization has distinct advantages in solving the Project Scheduling Problem for Resource Constrained through solving the examples in the relevant documents and analysis and comparison with the results in documents; the better satisfactory solution can be obtained, and meanwhile more scheduling schemes can be obtained to provide more choices for the decision maker of the project.

\section{References}

[1] J. Hu and Z. Gao, "Modules identification in gene positive networks of hepatocellular carcinoma using Pearson agglomerative method and Pearson cohesion coupling modularity", Journal of Applied Mathematics, vol. 2012, (2012).

[2] Y. Geng, J. Chen, R. Fu, G. Bao and K. Pahlavan, "Enlighten Wearable Physiological Monitoring systems", On-Body RF Characteristics Based Human Motion Classification Using a Support Vector Machine, (2015), pp. 99.

[3] X. Song and Y. Geng, "Distributed Community Detection Optimization Algorithm for Complex Networks", Journal of Networks, vol. 9, no. 10, (2014), pp. 2758-2765.

[4] K. Pahlavan, P. Krishnamurthy and Y. Geng, "Localization Challenges for the Emergence of the Smart World", Access, IEEE, vol. 3, no. 1, (2015), pp. 1-11.

[5] J. He, Y. Geng, Y. Wan, S. Li and K. Pahlavan, "A cyber physical test-bed for virtualization of RF access environment for body sensor network. Sensors Journal, IEEE, vol. 13, no. 10, (2013), pp. 38263836.

[6] Z. Lv, A. Tek and F. D. Silva, "Game on, science-how video game technology may help biologists tackle visualization challenges", PloS one, vol. 8, no. 3, (2013), pp. 57990.

[7] T. Su, W. Wang and Z. Lv, "Rapid Delaunay triangulation for randomly distributed point cloud data using adaptive Hilbert curve", Computers \& Graphics, vol. 54, (2016), pp. 65-74.

[8] J. Hu, Z. Gao and W. Pan, "Multiangle Social Network Recommendation Algorithms and Similarity Network Evaluation", Journal of Applied Mathematics, vol. 2013, (2013).

[9] S. Zhou, L. Mi, H. Chen and Y. Geng, "Building detection in Digital surface model", 2013 IEEE International Conference on Imaging Systems and Techniques (IST), (2012) October.

[10] J. He, Y. Geng and K. Pahlavan, "Toward Accurate Human Tracking: Modeling Time-of-Arrival for Wireless Wearable Sensors in Multipath Environment", IEEE Sensor Journal, vol. 14, no. 11, (2014) November, pp. 3996-4006. 
[11] Z. Lv, A. Halawani and S. Fen, "Touch-less Interactive Augmented Reality Game on Vision Based Wearable Device", Personal and Ubiquitous Computing, vol. 19, no. 3, (2015), pp. 551-567.

[12] G. Bao, L. Mi, Y. Geng, M. Zhou and K. Pahlavan, "A video-based speed estimation technique for localizing the wireless capsule endoscope inside gastrointestinal tract", 2014 36th Annual International Conference of the IEEE Engineering in Medicine and Biology Society (EMBC), (2014) August.

\section{Author}

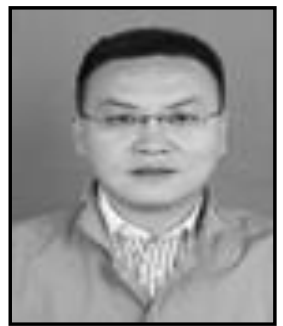

Yu Bo, received his Ph.D. in Management from Northwest A\&F University, China. He is currently a lecturer in the Department of Management and Technology at Xijing University. His research interest is mainly in the area of human resources management, agricultural economics \& management. He has published several research papers in scholarly journals in the above research areas. 